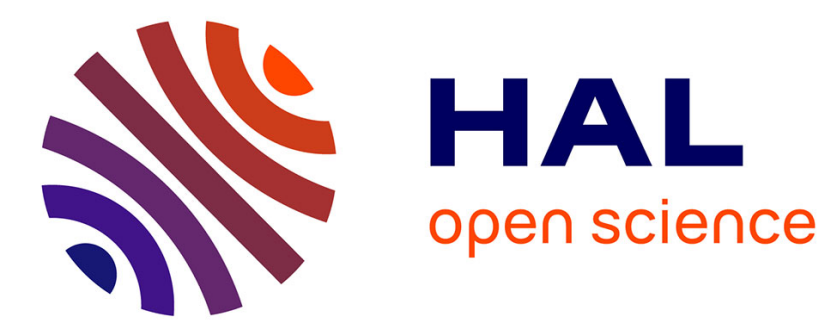

\title{
Experimental full field analysis for dynamic fractureof elastomer membranes
}

T Corre, M Coret, E Verron, B Leblé, F Le Lay

\section{To cite this version:}

T Corre, M Coret, E Verron, B Leblé, F Le Lay. Experimental full field analysis for dynamic fractureof elastomer membranes. International Journal of Fracture, 2020, 10.1007/s10704-020-00447-1 . hal03027053

\section{HAL Id: hal-03027053 \\ https://hal.science/hal-03027053}

Submitted on 26 Nov 2020

HAL is a multi-disciplinary open access archive for the deposit and dissemination of scientific research documents, whether they are published or not. The documents may come from teaching and research institutions in France or abroad, or from public or private research centers.
L'archive ouverte pluridisciplinaire HAL, est destinée au dépôt et à la diffusion de documents scientifiques de niveau recherche, publiés ou non, émanant des établissements d'enseignement et de recherche français ou étrangers, des laboratoires publics ou privés. 


\title{
Experimental full field analysis for dynamic fracture of elastomer membranes
}

\author{
T. Corre - M. Coret - E. Verron • B. \\ Leblé · F. Le Lay
}

Received: 6 October 2019 / Accepted: 4 April 2020

\begin{abstract}
Crack speed prediction in elastomer membranes under large strain (more than $150 \%$ ) remains a delicate question which necessitates coupled experimental-theoretical investigations. In order to compare experimental results with theoretical approaches but also to improve the understanding of dynamic crack propagation in soft materials, a crucial step is the measurement and analysis of kinematic and energetic fields in the material configuration during dynamic fracture. In this way, the proposed set-up consists in a two-camera set-up in order to perform digital image correlation during both quasi-static loading and dynamic fracture of the sample. The variety of kinematic and energetic fields that can be computed is illustrated. Finally, two studies highlighting the promises of full-field measurements in large strain fracture mechanics are presented: the computation of the dynamic $J$-integral and the observation of mechanical waves during high speed crack growth in elastomer membranes.
\end{abstract}

Keywords dynamic fracture · finite strain · elastomer · full-field measurements

\section{Introduction}

High speed fracture of rubber-like materials is a long lasting issue, as illustrated by past (Treloar, 1944a) and very recent (Moulinet and Adda-Bedia 2015) experimental studies devoted to the emblematic problem of the bursting balloon. Theoretically, crack growth in elastomers requires extension of the

T. Corre · M. Coret · E. Verron

Institut de Recherche en Génie Civil et Mécanique, UMR CNRS 6183, École Centrale de Nantes, France

E-mail: thomas.corre@ensta-paris.fr

T. Corre · B. Leblé · F. Le Lay

Naval Group Research, Technocampus Ocean, rue de l'Halbranne, 44340 Bouguenais, France 
standard framework of linear elastic fracture mechanics to both geometrical and material non-linearities. Rivlin and Thomas (1953) extended the Griffith (1921) theory of fracture to the case of elastomers, proposing an energy-based criterion for crack advance in material under large strain.

Since then, extensive studies have been devoted to the measurement of the energy release rate, first for quasi-static propagation (Rivlin and Thomas (1953); Greensmith (1963); Yeoh (2001)) but also during dynamic propagation from low rate (Kadir and Thomas (1981); Gent (1996); Lake et al. (2000)) up to high crack speed (Lake et al. (2000); Morishita et al. (2016)). These studies focus on the influence of material parameters or crack speed on the energy release rate, which is computed with analytical formulas derived for simple specimen (Rivlin and Thomas, 1953). Thus, if the crack speed is recognized as one of the main feature of interest, only few studies investigate its evolution in highly stretched membranes (Stevenson and Thomas (1979); Gent and Marteny (1982)) and even less for supersonic crack speed (Petersan et al. (2004); Chen et al. (2011)). In this case, the validity of the historical approach is not well-established (Gent and Marteny, 1982).

Fast cracks in elastomers are mostly observed in biaxially strained membranes. In this case, 2D digital image correlation (DIC) turns out to be a very suitable technique to measure kinematic fields. Indeed, with the plane stress assumption and the incompressibility of elastomers, the out-of-plane strain can be directly computed from 2D strain fields measured on the surface. Improvements in the DIC technique have extended its applicability to large strain (Chevalier et al., 2001), in particular for strain fields around static cracks in rubber (Rublon et al., 2014, Caimmi et al., 2015, Qi et al., 2019). For moving cracks, many optical techniques have been developed for fragile materials, from photoelasticimetry (Dally, 1979) or caustics (Washabaugh and Knauss, 1994) to DIC with high frequency cameras (Chao et al., 1998; Kirugulige and Tippur, 2009) for linear elastic fracture mechanics. In the case of elastomers, which combine high speed cracks and finite strain, very few studies consider full field measurements during dynamic crack growth. Zhang et al. (2009) measured the velocity field around a supersonic crack in natural rubber with particle image velocimetry. To the authors' knowledge, the first full-field measurements around a fast crack $\left(2 \mathrm{~m} . \mathrm{s}^{-1}\right)$ in a brittle elastomer (polyacrylamide gels) have been recently proposed by Livne et al. (2010) who track a passive tracer field printed on the gel to measure displacement and strain fields, the nominal stretch in the membrane being up to $13 \%$.

Yet experimental analysis of dynamic fracture of elastomers is still in its infancy, no study has investigated cracks growing under complex loading. In this paper, we propose to benefit from the improvement of high-speed imaging to measure all kinematic fields during crack growth, aiming for an effective procedure for crack growth in initially inhomogeneous strain fields. In particular, monitoring both deformation and fracture of the membrane allows the push-back of kinematic fields in the reference configuration, the first necessary step for a complete mechanical analysis of the phenomenon. This method addresses various issues in large strain dynamics, such as the choice of the 
relevant configuration to analyse the problem and the feasibility of measuring fracture mechanics quantities. In the following, the experimental method is thoroughly presented in section 2 . Then, the crack growth is characterised in section 3 with, as a main result, the various full-fields we measure, from kinematic to energetic fields. These mechanical fields allow for the direct computation of the $J$-integral and the discussion on the energy flux toward the crack tip provided in section 4 As a supplementary investigation, the observation of mechanical waves during crack growth further illustrates the relevance of the method. Finally, section 5 concludes both on the experimental technique itself, and its specific assets for dynamic fracture of elastomers.

\section{Set-up for full-field measurements during high speed crack growth}

\subsection{Sample and material}

Experiments are carried out with "pure shear" specimens (Yeoh, 2001), which are widely used for dynamic crack growth, sometimes with prescribed stretch ratio in both vertical and horizontal directions (Gent and Marteny, 1982; Deegan et al. 2001, Morishita et al., 2016)). Such samples present the advantage to provide an analytical expression of the energy release rate (Rivlin and Thomas. 1953), but they also offer a wide area with homogeneous strain field that allows the observation of steady state crack growth over a long distance. The pure shear sample is a long and thin band of material; here, its dimensions are $200 \times 40 \times 3 \mathrm{~mm}^{3}$. In order to avoid slippage during loading, samples are moulded with two cylindrical bulges (diameter $15 \mathrm{~mm}$ ) at the edge of their long sides to fit into specially designed grips. In the following, the "working area" of samples will refer to the above mentioned thin band situated between the bulges. So, the width/height ratio of the working area is 5 ; it is the smallest ratio that satisfies pure shear conditions, i.e. neither extension nor compression in the width direction at the centre of the specimen (Yeoh, 2001). Nevertheless, with such a small geometrical ratio, areas of complex strain field on each side of the specimen are growing rapidly with the prescribed global stretch ratio, reducing the size of the homogeneous pure shear deformation area. In the present study, this limitation will be overcome by the direct measurement of the strain fields with the DIC technique, dispensing with the hypothesis of pure shear state. For DIC measurements, a random black speckle pattern is sprayed on the undeformed surface of the specimens; the material being naturally clear and slightly translucent, it offers a good contrast with the black paint drops under vivid see-through lightning.

The material is a commercial polyurethane made of toluene diisocyanate and polyether. It is assumed homogeneous, isotropic, and incompressible. Then its density $\rho_{0}$ does not depend on the deformation and is equal to $1044 \mathrm{~kg} \cdot \mathrm{m}^{-3}$. To determine its mechanical response, uniaxial tension experiments with constant true strain rate of $10^{-3} \mathrm{~s}^{-1}$ are conducted and the corresponding nominal 
stress vs. stretch ratio response is shown in Figure 1. They were carried out

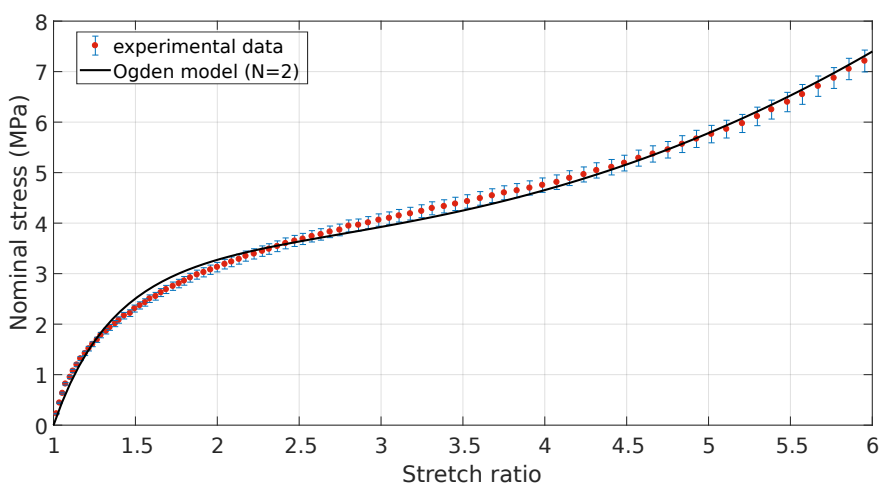

Fig. 1 Nominal stress vs. stretch ratio in uniaxial tension at constant true strain rate $\left(10^{-3} \mathrm{~s}^{-1}\right)$. Comparison between experimental data and the 2-term hyperelastic Ogden model.

with an INSTRON 5584 tensile machine with a $1 \mathrm{kN}$ load cell. To reach high stretch ratios, the tensile sample also have cylindrical bulges. The tests are monitored with a camera (AVT Prosilica GT 6600, lens Zeiss 2/100 ZF) and the strain is measured on pictures by a point tracking method. The main source of inaccuracy is the variability in the section of the sample, explaining the error bars in Fig.1. Constant strain rate is prescribed by applying a displacement varying exponentially with time at one grip (Petiteau et al., 2013$)$. The value of $10^{-3} \mathrm{~s}^{-1}$ is a compromise between prescribing a very low strain rate, reaching high stretch ratios, and the capabilities of the machine. In the following, the stress-strain curve measured at this strain rate is considered as the quasi-static response of the material. Note that with classical uniaxial tension experiment at constant crosshead speed (displacement varying linearly, the true strain rate is not constant), the strain at failure was measured at about $800 \%$.

For further computation of stress and strain energy, experimental data are fitted with a 2-term Ogden hyperelastic model (Ogden, 1972),

$$
P=\sum_{i=1}^{2} \mu_{i}\left(\lambda^{\alpha_{i}-1}-\lambda^{-\frac{\alpha_{i}}{2}-1}\right)
$$

where $P$ is the nominal stress, $\lambda$ is the stretch ratio, and $\left(\mu_{i}, \alpha_{i}\right)_{i=1,2}$ are the material parameters. Their best fit with experimental data gives

$$
\mu_{1}=6.188 \times 10^{-2} \mathrm{MPa}, \alpha_{1}=3.456 ; \mu_{2}=-12.73 \mathrm{MPa}, \alpha_{2}=-0.478 \text {. }
$$

Note that this model ignores any viscous effects induced by large strain rate. As experimentally observed (with the classical data of Treloar (1944b) for example), the response of elastomers in uniaxial tension and pure shear are very 
close (see Ogden (1972), and Marckmann and Verron (2006) among others. Then, because the deformation state in the cracked membrane is always between these two types of deformation, our fit is assumed relevant for the present problem.

\subsection{Loading conditions and procedure}

Practically, the experimental procedure is split into two steps as depicted in Figure 2, First the virgin specimen, i.e. without crack, is stretched to the

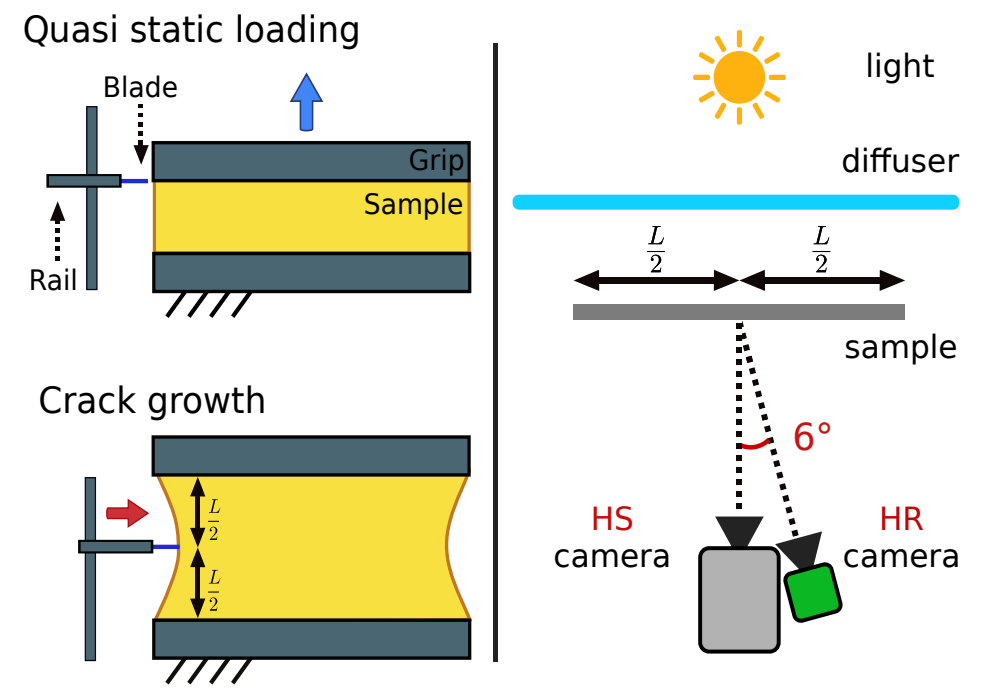

Fig. 2 Left: the two step procedure - loading of the sample and crack initiation with a blade. Right: Scheme of the set-up with the relative location of the two cameras (top view).

prescribed stretch ratio. Second, a manual cut with a razor blade initiates the crack at the centre of one edge. More specifically:

- During the first step, the specimen is stretched in the tensile machine until the displacement corresponds to the prescribed stretch ratio, the crosshead speed being set to $20 \mathrm{~mm} / \mathrm{min}$. This part of the experiment is recorded with a high resolution camera STEMMER AVT GT 6600 (29 Mpix), referred to as HR camera in the following. The spatial resolution is about 0.04 $\mathrm{mm} / \mathrm{pix}$ and the frame rate is $0.5 \mathrm{~Hz}$.

- The second step starts with the cut of the crack as illustrated in Figure 3 The cut is made with a blade fixed on a sliding rail that is operated manually. Crack propagation is recorded with a high speed camera Photron SAZ, referred to as HS camera throughout the rest of the paper. The frame rate is adapted for each test, and its maximum value is $40,000 \mathrm{~Hz}$ for full resolution $(1024 \times 1024)$. The spatial resolution is about $0.2 \mathrm{~mm} / \mathrm{pix}$. It is to 


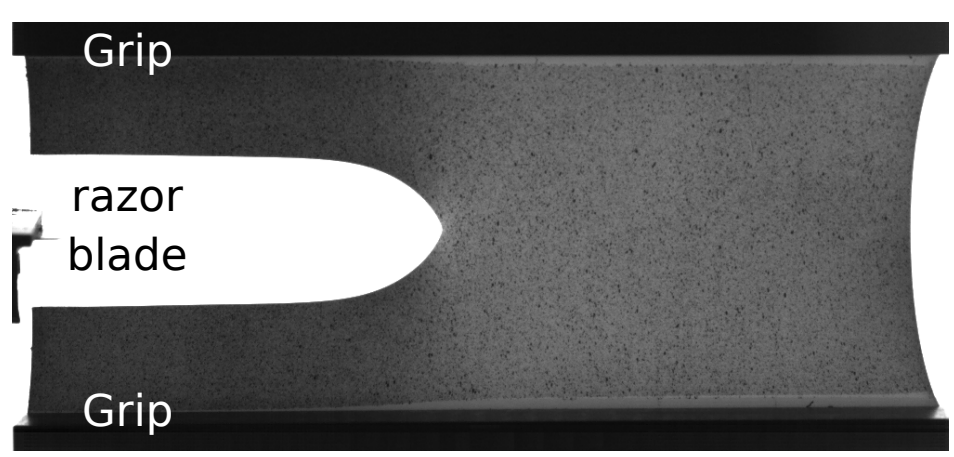

Fig. 3 Crack growth in pure shear specimen. Prescribed stretch ratio : 2. Instantaneous crack velocity: $33.1 \mathrm{~m} . \mathrm{s}^{-1}$. HS camera: $20000 \mathrm{fps}$, shutter speed 1/160000 s. Note the random black speckel sprayed on the specimen.

note that the cut is made while the crosshead is still in motion. However, due to the short duration of this second step, i.e. between 16 and $300 \mathrm{~ms}$, it is assumed fixed.

\subsection{Full-field measurements}

\subsubsection{Measurement of the displacement field}

The commercial software Vic-2D is used to measure the displacement field by DIC during the two steps of the experiments. This software uses a local DIC method based on matching $n \times n$ pixel squares of a regular grid. This grid serves as the FEM support for our analysis as explained in the following. Matching is defined by the conservation of the optical flow between two images whose random speckle is the digital signature (Sutton et al, 2009). Due to both thickness of the specimens and in-plane loading conditions, the problem is assumed in a plane stress state such that membrane thinning is easily computed from in-plane displacements and the incompressibility assumption.

As mentioned above, pictures are recorded with two different cameras, resulting in two independent measures of the displacement field. The displacement field measured during the second step does not take the loading step into account. To compute the total displacement field, data from HS camera (second step) are combined with those from HR camera (first step) and projected onto HS images. Adopting a continuous kinematic description, the first image of the HR camera corresponds to the stress free reference configuration of the sample, where the coordinates of the material points are denoted $\mathbf{X}$. During loading and crack growth steps, the coordinates of the material points are denoted $\mathbf{x}_{l}$ and $\mathbf{x}_{c}$ respectively. Along the first step, the HR camera provides the displacement field directly from the definition:

$$
\mathbf{u}_{l}=\mathbf{x}_{l}-\mathbf{X}
$$


During crack growth, the HS camera measures the displacement from the end of the first step:

$$
\mathbf{u}_{c}=\mathbf{x}_{c}-\mathbf{x}_{l}
$$

which leads to the total displacement field at any time $t$ after crack initiation at $t_{c}$ :

$$
\mathbf{u}(t)=\mathbf{u}_{l}\left(t_{c}\right)+\mathbf{u}_{c}(t)
$$

Practically, the DIC software computes the position of the nodes of a grid covering the area of the specimen; but the grids of the two cameras do not coincide. However, with a low crosshead speed, it is assumed that the last picture of the HR film and the first picture of the HS film, just before cutting the sample, represent the same mechanical state of the sample and therefore correspond to the same displacement field. Between these two pictures, the displacement field $\mathbf{u}_{l}$ measured on the HR grid can be projected onto the HS grid $\left[\mathbf{x}_{c}\right]$ (in the following, the projection of a given continuous field $*$ onto the HS grid is denoted $[*])$. To do so, the grids of the corresponding images are superimposed (homothetic transformation and positioning from specific points of the sample) and the displacement $\mathbf{u}_{l}$ is projected on the nodes $\left[\mathbf{x}_{c}\right]$ (linear interpolation from the first grid mesh). The grid from the HR camera is about $30 \%$ more dense than the HS grid, which favours the precision of the projection. Then, the total displacement $\mathbf{u}$ is computed at the nodes $\left[\mathbf{x}_{c}\right]$ thanks to Eq. (5), and the positions of $\left[\mathbf{x}_{c}\right]$ in the reference configuration, $\left[\mathbf{X}_{c}\right]$, are

$$
\left[\mathbf{X}_{c}\right]=\left[\mathbf{x}_{c}\right]-\mathbf{u}\left(\left[\mathbf{x}_{c}\right]\right)
$$

Remark 1 On the one side, the reference configuration is defined here as the undeformed and uncracked sample at the beginning of the loading step. The grid $\left[\mathbf{X}_{c}\right]$ maps the sample in this configuration and the initial rectangular shape of the working area is recovered. On the other side, the material configuration refers to an abstract stress and strain free configuration of the cracked sample (Maugin, 1995). In this configuration, the crack is a line that gets longer with time. So, the material configuration only differs from the reference configuration by the presence of the crack: $\mathbf{X}_{\mathbf{c}}$ provide the position of material points in both reference and material configurations. In the following, the term material configuration will be used when mentioning mechanical fields projected onto the $\left[\mathbf{X}_{c}\right]$ grid.

Remark 2 The two cameras cannot be placed right in front of the sample at the same time: we have chosen to adjust the HS camera and then to set the HR camera as close as possible but with a little angle (see Fig 2 right). This angle, computed from the recorder pictures, equals $6.5 \pm 0.5^{\circ}$. It leads to an error on the horizontal displacement measured during the first step of approximately 0.6 $\%$ and does not impact the vertical displacement measurements. 


\subsubsection{Space and time derivatives}

Once the material configuration and the displacement field are determined, the deformation gradient $\mathbf{F}$ is computed for any image of the second step (crack growth):

$$
\mathbf{F}=\mathbf{I}+\frac{\partial \mathbf{u}}{\partial \mathbf{X}_{c}}
$$

where $\mathbf{I}$ is the second order $3 \times 3$ identity tensor. For each image, Eq. 77 is derived using a finite element scheme. The material configuration is meshed with linear triangular elements whose nodes are defined by the positions of the points of the $\left[\mathbf{X}_{c}\right]$ grid provided by the DIC, as shown in Figure 4(a). Thus, any strain field can be computed for every frame. The discontinuity induced by the crack is not disturbing because the elements crossed by the crack are suppressed (DIC breaks down close to the crack tip, as shown in Fig. 4(b) and discussed in section 3.3 .

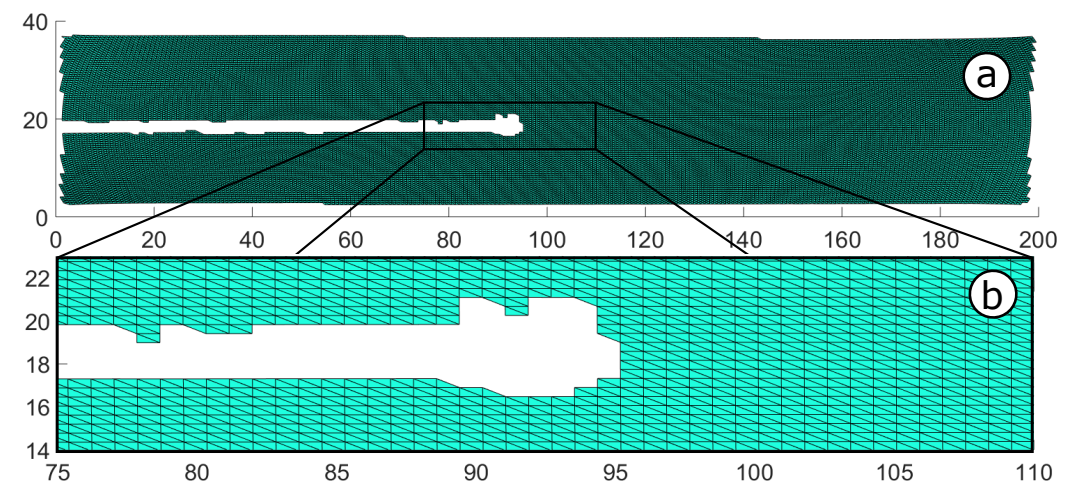

Fig. 4 (a) Mesh in the material configuration and (b) zoom around the crack tip where DIC breaks down (white area). The scales are in $\mathrm{mm}$.

Finally, thanks to the high frame rate of the HS camera, time derivatives of any mechanical field can be accurately computed during crack growth: the time evolution of any strain field is given by the series of frames during crack growth. In practice, the evolution of fields to be derived at a given node is first fitted by a polynomial of degree 2 over 5 time steps, then the time derivative is computed with this polynomial in order to reduce the noise induced by numerical derivation. Note that first and second time derivatives of the displacement field naturally give velocity and acceleration fields, respectively; whereas the time derivative of $\mathbf{F}$ provides strain rate tensors. 


\section{Results for dynamic crack growth under large strains}

\subsection{Global analysis of crack propagation}

After cutting, the crack freely grows, faster than the blade. Time evolution of crack length is measured from the remaining length of the sample in the deformed configuration; an example of such evolution is drawn in Figure 5(a). The high contrast of pictures allows measurement of the crack tip position
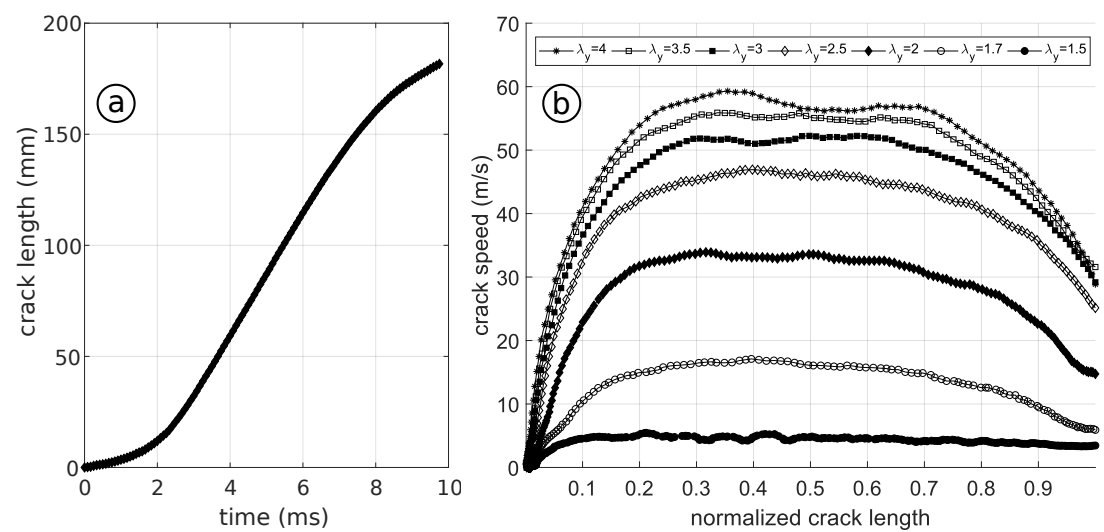

Fig. 5 (a) Time evolution of crack length in a sample with a prescribed stretch ratio of 2. Time origin is arbitrary. (b) Crack speed vs.normalized crack length, i.e. distance travelled by the crack divided by sample width, for different prescribed stretch ratios $\left(\lambda_{y}\right)$.

with a simple binarization. Then, the crack speed is computed using a local polynomial fit of the time evolution of the remaining length (degree 2 over 5 time steps). The corresponding results for different prescribed stretch ratios are shown in Fig 5 (b). All curves are similar and three stages are exhibited: the crack speed rises, then reaches a plateau, and finally decreases. As expected, the pure shear sample leads to a steady state propagation of the crack which corresponds to the plateau; it is due to the wide homogeneously deformed area in the middle of the sample.

Steady state growth Figure 6(a-d) presents some possible shapes of the crack tip opening during steady state crack growth for different stretch ratios; Fig 6(e) depicts the corresponding crack speeds. The evolution of the steady state speed, i.e. a strong increase at low stretch ratios followed by a milder slope, is very similar to what has been reported for natural rubber by Gent and Marteny (1982), and Zhang et al. (2009). The change of the crack tip shape, from a parabolic tip in Fig]6(a-b) to a wedge-like crack opening in Fig]6(c-d), has been also observed by Zhang et al. (2009), and Morishita et al. (2016). In every case the crack growth should be considered dynamic, the slowest crack still growing at $4.71 \mathrm{~m} . \mathrm{s}^{-1}$. But for a stretch ratio higher than 2, the crack 


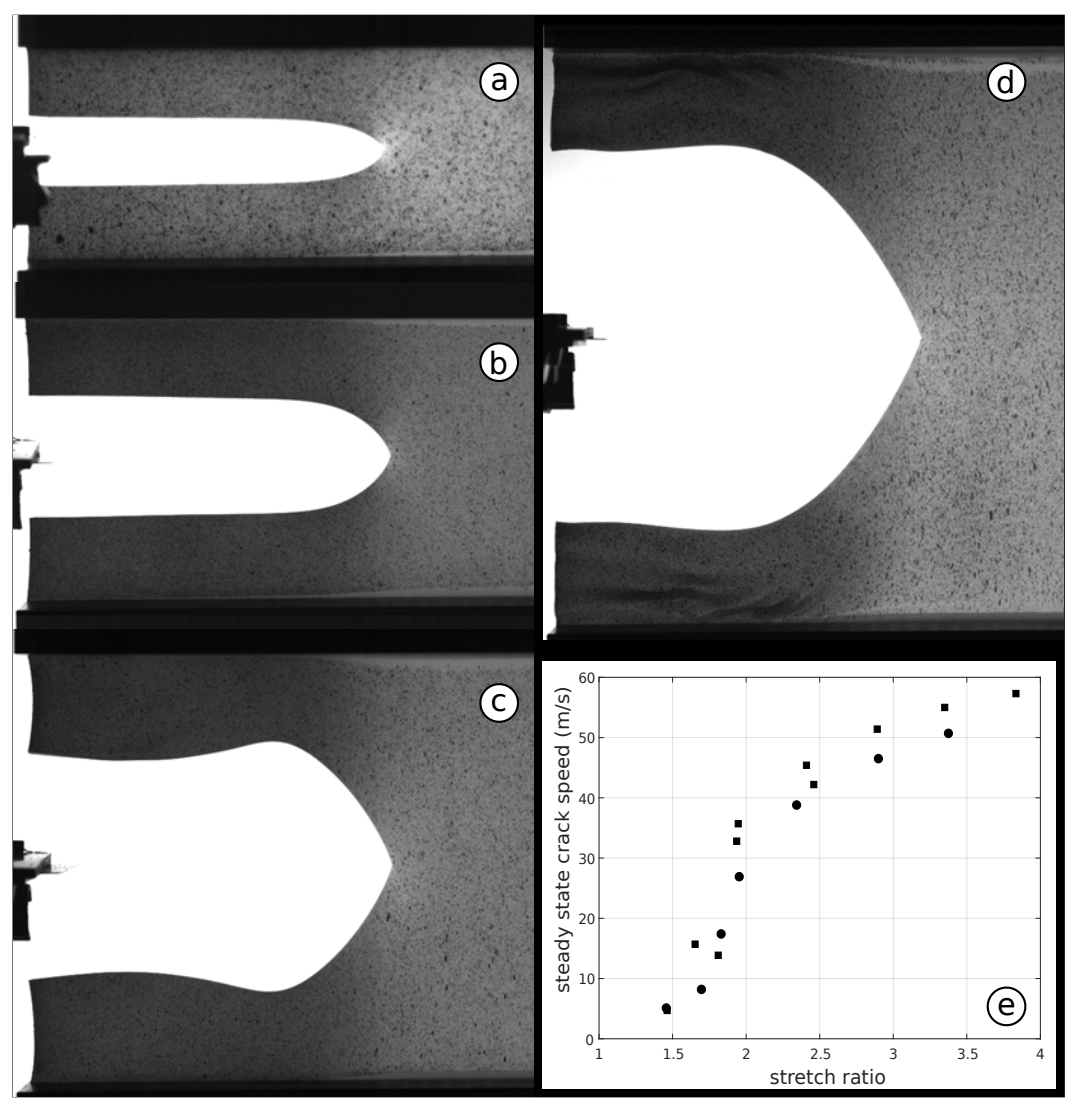

Fig. 6 (a-d) Shape of the crack tip during steady state propagation. The initial stretch ratios are (a) 1.46 , (b) 1.94 , (c) 2.89 and (d) 3.84 . The corresponding crack speeds are (a) 4.71, (b) 32.8 , (c) 51.4 and (d) $57.3 \mathrm{~m} . \mathrm{s}^{-1}$. (e) Steady state crack speed vs. prescribed stretch ratio (circles and squares refer to different series of tests with different material batches)

lips are retracting fast enough to induce compression of the membrane. For even higher stretch ratios, some out-of-plane displacement and even wrinkles induced by buckling are observed (Fig $6(\mathrm{~d}))$. As expected, the DIC is then functioning only before the crack tip arrival.

\subsection{Local mechanical fields}

For moderate initial stretch ratios, mechanical fields can be computed on the whole working area. To illustrate the method and allow comparability, all the fields shown in this section are issued from a test with a stretch ratio of $1.936 \pm 0.002$ ( 2 was prescribed). The instant chosen corresponds to the picture in Fig 3 where the crack is growing at $33 \pm 0.5 \mathrm{~m} . \mathrm{s}^{-1}$. In addition, sample sizes are indicated by the vertical and horizontal axes, graduated in $\mathrm{mm}$. 
Mechanical configurations The first field obtained from DIC is the displacement field. As an example, Figure 7(a) shows the horizontal displacement field in the deformed configuration. As explained above, the material configuration can be retrieved and the same field can be drawn in this configuration (Fig $7(b)$ ). In the deformed configuration, the black cross is the position of the crack tip measured directly on the picture (see Fig 3). The set-up cannot provide displacement measurements at less than $2 \mathrm{~mm}$ away from the crack tip (depending on the picture). Then, in the material configuration, the white line refers to the elements where DIC broke down along the crack path.
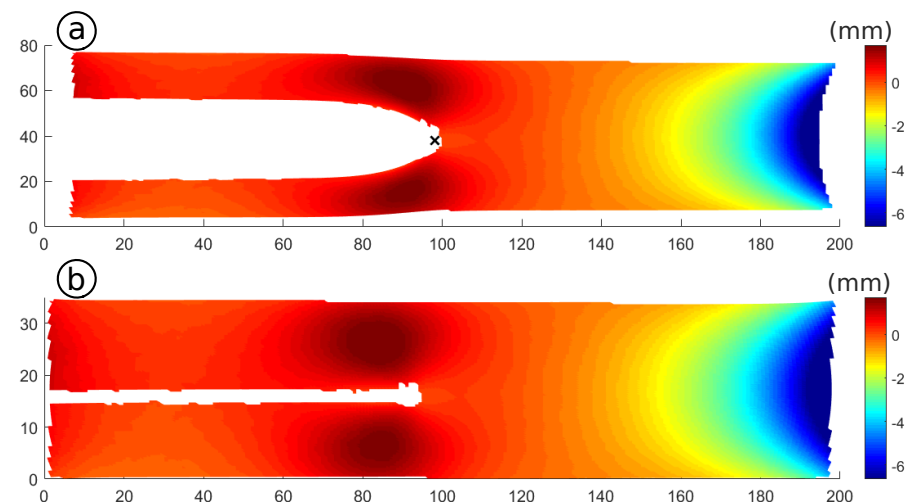

Fig. 7 Horizontal displacement field during crack growth (a) in the deformed configuration and (b) projected onto the material configuration. The black cross in the deformed configuration (a) marks the actual crack tip position.

Time derivatives The high working frequency of the high speed camera allows the accurate derivation of the velocity field during crack growth. Figure 8 presents both horizontal and vertical components of the velocity field, highlighting the trajectory of material points above the crack tip. The method is precise enough to capture both high vertical retraction velocities behind the crack tip $\left(\simeq 30 \mathrm{~m} . \mathrm{s}^{-1}\right)$ and slow horizontal movements.

Spatial derivatives Knowing the reference configuration, any strain field can be computed thanks to the deformation gradient. Figure 9(a) shows the GreenLagrange strain tensor component $E_{y y}$, with $\mathbf{E}=\frac{1}{2}\left(\mathbf{F}^{T} \mathbf{F}-\mathbf{I}\right)$. Combined with the time derivative technique presented above, the strain rate tensor is also available: it is computed from the time derivative of the deformation gradient $\dot{\mathbf{F}}$,

$$
\mathbf{D}=\operatorname{sym}\left(\dot{\mathbf{F}} \mathbf{F}^{-1}\right)
$$

One of its component is shown in Fig $9(\mathrm{~b})$. 

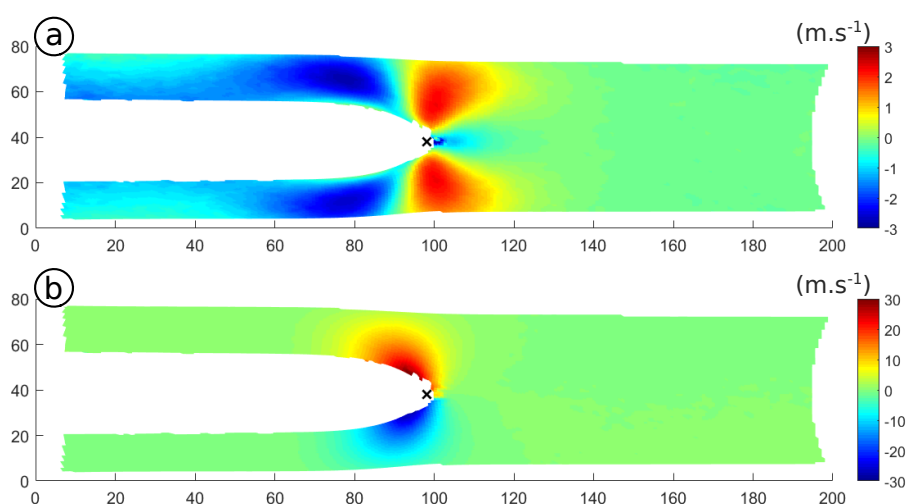

Fig. 8 Velocity field in the deformed configuration $\left(\mathrm{m} \cdot \mathrm{s}^{-1}\right)$ : (a) horizontal velocity, (b) vertical velocity. The black cross shows the position of the crack tip, moving at $33.1 \mathrm{~m} . \mathrm{s}^{-1}$ in this frame.

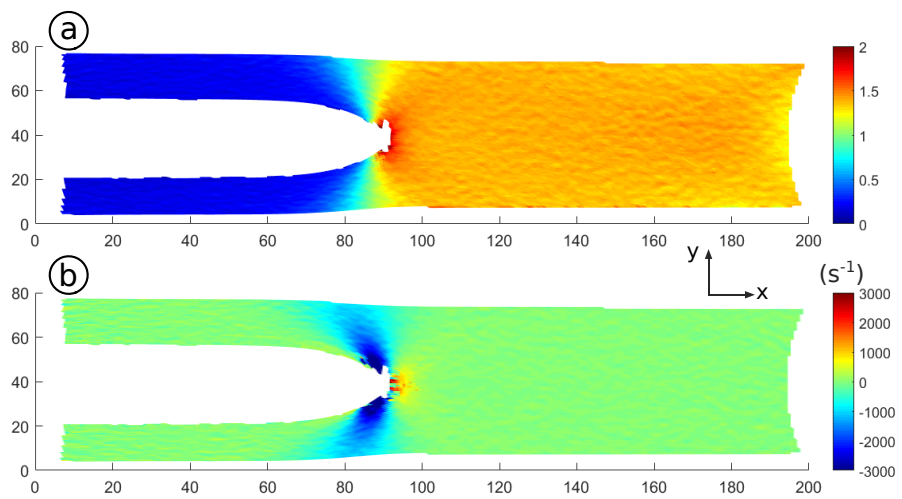

Fig. 9 In the deformed configuration: (a) Green-Lagrange strain tensor component $E_{y y}$, (b) strain rate tensor component $D_{y y}$.

Energy fields With the velocity field, and assuming the incompressibility of the elastomer, it is easy to compute the kinetic energy density field during the fracture process as shown in Figure 10(a). The use of the constitutive equation allows the calculation of the stress field. Here, the chosen model (hyperelastic 2-term Ogden) does not account for the viscous response of the material. Then, it lacks accuracy where the material experiences high strain rates, especially in front of the tip and behind where the retraction velocity is maximum. However, the model remains relevant to evaluate the stored energy, namely the strain energy density per unit of undeformed volume, depicted in Fig 10(b). The availability of these energy fields paves the way for an energetic analysis of crack growth from an experimental point of view. As an example, Fig.11 highlights the predominance of strain energy over kinetic energy in the sample. In general, the strain energy density mostly dominates the kinetic energy density. As expected, the non-zero kinetic energy is located behind the 

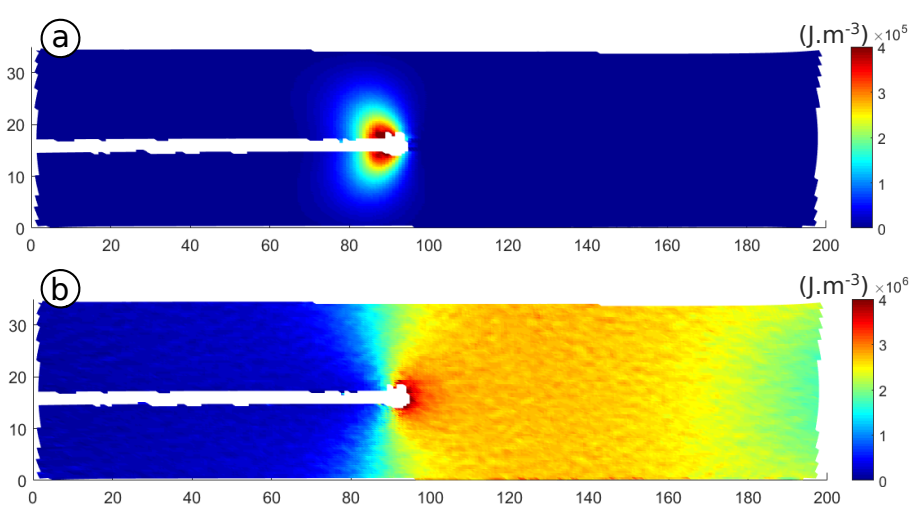

Fig. 10 Energy fields projected onto the material configuration during crack growth at $33.1 \mathrm{~m} \cdot \mathrm{s}^{-1}\left(\mathrm{~J} . \mathrm{m}^{-3}\right)$. (a) Kinetic energy density. (b) Strain energy density.

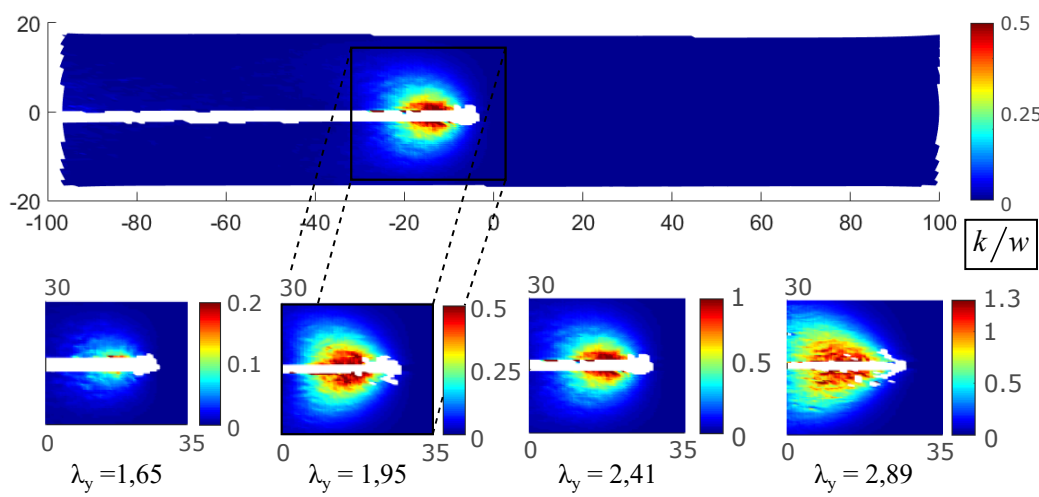

Fig. 11 Ratio between kinetic and strain energy densities for four prescribed stretch ratios. The corresponding steady state crack speeds in ascending order are 15.7, 32.8, 45.4, and $51.4 \mathrm{~m} . \mathrm{s}^{-1}$. Top panel is computed from the fields of Fig 10

crack tip where crack lips are retracting, as shown in Fig.8(b). Locally, both energy densities admit the same order of magnitude only for the highest crack speeds.

\subsection{Robustness of the measurement protocol}

Repeatability The repeatability of successive experiments is satisfactory. Indeed, the test with a prescribed stretch ratio of 3 has been repeated 3 times, providing a crack growth speed in the steady state regime of $46.2 \pm 0.5 \mathrm{~m} . \mathrm{s}^{-1}$. The repeatability decreases for stretch ratios from 1.7 to 2 because of the strong influence of a small variation in the actual prescribed stretch ratio on crack speed (see Fig.5). Moreover, discrepancy is observed between the two batches of material used (and tested at different times), as highlighted in 
Fig 5 Whether this discrepancy is due to the formulation of the material or experimental conditions, e.g. temperature, is not clear yet. The cutting blade is moved manually, but neither its speed nor its angle has a noticeable influence on the free crack growth. The speed of the cutting blade, measured afterwards on the films, is maintained between $0.3 \mathrm{~m} \cdot \mathrm{s}^{-1}$ and $0.7 \mathrm{~m} \cdot \mathrm{s}^{-1}$.

Precision Firstly, field measurements predominantly depend on the displacement field computed by the DIC software. To estimate the precision of this primary measurement, the software provides the confidence interval for the position of each node. This interval varies significantly over one frame, from $4 \times 10^{-3} \mathrm{~mm}$ far from the crack to $10^{-2} \mathrm{~mm}$ just beyond (values from the HS camera only). However, in spite of the knowledge of the uncertainties in the measured displacement field, we are unable to reliably estimate the precision of the final deformation fields, because of the numerical projection step described in section 2.3.1. Secondly, the precision of the velocity field can be estimated by the noise observed in areas of the sample supposedly at rest at the beginning of crack growth. With this technique, we estimate that the velocity field is measured with a precision of about $0.05 \mathrm{~m} . \mathrm{s}^{-1}$. Finally, in spite of the intrinsic precision of the measurements, the present set-up cannot provide information at less than about $2 \mathrm{~mm}$ away from the crack tip (see Fig.7(a) for instance). The size of the blank zone around the crack increases with the prescribed stretch ratio for three main reasons. First, in front of the crack tip, the material is so stretched that too much light passes through the thin membrane and floods the camera (see-through lightning in Fig 22). Second, just beyond the crack tip, the high level of shear and the proximity of free edges complicates the correlation. And third, the high retraction speed of both lips immediately after the crack tip can locally blur the pictures and expel some paint drops.

\section{First applications of the method}

\subsection{Measuring the dynamic energy release rate}

In the classical approaches of fracture, especially in the case of linear elastic fracture mechanics (LEFM), the crack speed $V$ is predicted by equating the instantaneous energy release rate $G$ with its critical value for a given crack speed $\Gamma(V)$, i.e. the fracture energy (Freund, 1998). Practically, predicting crack growth requires the determination of the curve $\Gamma$ vs. $V$, assumed to be a material parameter for a given elastomer (Greensmith and Thomas, 1955). The $J$-integral provides an efficient way to compute the energy release rate (Rice, 1968). However, the use of such analytical formulas in an experimental context is challenging and requires full-field measurements techniques. There have been very few attempts for elastomers (large strain). Nonetheless Caimmi et al. (2015) have used DIC to compute the $J$-integral around a static crack in a rubber sheet, and Livne et al. (2010) have measured it around a rapid 
crack $\left(2 \mathrm{~m} . \mathrm{s}^{-1}\right)$ in a brittle elastomer under moderate strain level. Using our full-field measurements technique, we now question the possibility to measure the energy release rate in a very general case which combines large strain, non-linear elasticity, and high speed crack growth at non-constant speed.

The elastodynamic version of the $J$-integral was proposed by Atkinson and Eshelby (1968), and Freund (1972), and extended to the non-linear case (both geometric and material) by Gurtin and Yatomi (1980). It is based on the computation of the flux of mechanical energy entering a contour encircling the crack tip in the material configuration. For a $2 \mathrm{D}$ problem with a crack growing along $\mathbf{e}_{1}$ at the speed $\mathbf{V}_{0}$ in the material configuration, the flux entering the contour $\Sigma$ of outward normal unit vector $\mathbf{N}$ is:

$$
\Phi(\Sigma)=\int_{\Sigma}\left[(w+k) \mathbf{V}_{0}+\mathbf{P}^{T} \cdot \frac{\partial \mathbf{u}}{\partial t}\right] \cdot \mathbf{N} \mathrm{d} \Sigma,
$$

where $w$ is the strain energy density per unit of volume in the reference configuration, $k$ is the kinetic energy density, $\mathbf{u}$ the displacement field, and $\mathbf{P}$ the first Piola-Kirchhoff stress tensor (adapted from Freund (1998) Eq. (5.2.7)). Making use of the incompressibility of the material, the kinetic energy density is defined as follows,

$$
k=\frac{1}{2} \rho_{0}\left(\frac{\partial \mathbf{u}}{\partial t}\right)^{T} \cdot \frac{\partial \mathbf{u}}{\partial t} .
$$

The $J$-integral corresponding to the energy flux in Eq. 9 is then defined by considering a contour that shrinks toward the crack tip (adapted from Freund (1998) Eq. (5.3.2)):

$$
G=\lim _{\Sigma \rightarrow 0}\left(\frac{\Phi(\Sigma)}{\left\|\mathbf{V}_{0}\right\|}\right) .
$$

Contrary to the quasi-static case, the integral Eq. (9) is not path-independent in general. However, for steady-state crack growth, it is path-independent and the computation of the limit in Eq. (11) is not necessary (Moran and Shih, 1987). This particular case will be considered in the following and illustrated by an experiment with a prescribed stretch ratio of 2 in which the steady-state speed of the crack is $33 \mathrm{~m} \cdot \mathrm{s}^{-1}$.

Method Direct observation of crack propagation provides the crack tip kinematics in the laboratory frame. In the context of finite strain, retrieving the position and speed of the tip in the material frame requires some caution and at least two hypotheses. First, we assume that the position of the tip in the laboratory frame can be recovered from its position in the deformed configuration (denoted $\mathbf{x}_{c t}(t)$ ): there is no movement of the matter on the path of the crack. Experimentally, this assumption is verified except in the last millimetres of the crack path where the last portion of the sample moves because of the approaching crack and wave reflection. Second, we suppose that the displacement at the tip induced by the presence of the crack, $\mathbf{u}_{c}\left(\mathbf{x}_{c t}(t)\right)$, is small compared to the displacement induced by the loading step $\mathbf{u}_{l}\left(\mathbf{x}_{c t}(t)\right)$. 
Explicitly, with $\mathbf{X}_{c t}(t)$ and $\mathbf{x}_{c t}(t)$ the coordinates of the material point where the crack tip stands in the material and current configuration, this hypothesis reads:

$$
\mathbf{x}_{c t}(t)=\mathbf{X}_{c t}(t)+\mathbf{u}_{l}\left(\mathbf{x}_{c t}(t)\right)+\mathbf{u}_{c}\left(\mathbf{x}_{c t}(t)\right) \approx \mathbf{X}_{c t}(t)+\mathbf{u}_{l}\left(\mathbf{x}_{c t}(t)\right) .
$$

The symmetry of the crack opening in the case of a straight horizontal propagation in mode I imposes vertical component of $\mathbf{u}_{c}\left(\mathbf{x}_{c t}\right)$ to be null. However, the need for such hypothesis arises from the finite strain framework in which the component of $\mathbf{u}_{c}\left(\mathbf{x}_{c t}(t)\right)$ in the direction of the crack propagation is not strictly zero (Long et al., 2011, Goldman Boué et al., 2015). Combining these two assumptions allows the estimation of $\mathbf{u}_{l}\left(\mathbf{x}_{c t}(t)\right)$ (linear interpolation of the displacement field before crack growth at points $\mathbf{x}_{c t}$ ) and the derivation of the crack speed in the material frame:

$$
\mathbf{V}_{0}=\frac{\partial \mathbf{X}_{c t}(t)}{\partial t}
$$

The speed computed with Eq. (13) differs from the crack speed computed in section 3 principally close to the edges, where the horizontal stretch ratio is far from 1. Marder (2006) uses directly the deformation gradient of the loading step to compute the material crack speed from the "current" crack speed. In our case, the two techniques provide similar results within the experimental precision.

As aimed, all fields involved in Eq. (9) are available through the analysis presented above. Considering the large number of hypotheses required, the integration method is not the main source of uncertainties. This method is illustrated in Figure 12 and can be summarised as follow:

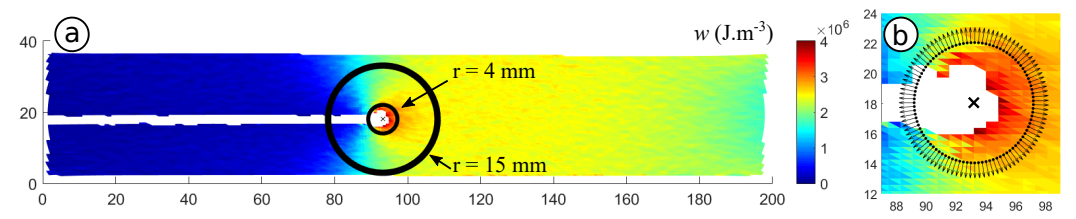

Fig. 12 (a) Smallest and largest contours used to compute the energy flux integral, centred on the crack tip location in the material configuration (radii in $\mathrm{mm}$ ). (b) Close-up on the $4 \mathrm{~mm}$ contour. The field is the strain energy density.

- The energy flux is computed on various circular domains around the crack tip in the material configuration, as shown in Figure 12(a). Note that the contours are independent from the mesh and defined analytically.

- As illustrated in Fig.12(b), some elements are missing in the integration zone around the tip: the lack of data in the $2 \mathrm{~mm}$ zone around the crack tip is overcome by considering contours with radii larger than this value. 
- Mechanical fields involved in Eq. (9) are computed in each element: it leads to constant value over the element because of the linear triangular finite element mesh, or it is interpolated at its barycentre for speed and displacement fields.

- The contour is discretized and the outward normal vector computed analytically at each node. The value of the vectorial fields in Eq. (9) are computed at each node of the contour from the corresponding value in the underlying element.

- The scalar product with the outward normal vector in Eq. (9) is computed at each node of the contour, and the resulting curve is integrated along the contour.

Unavoidably, data are not available on a small part of the contour behind the crack tip in the neighbourhood of crack lips (see Fig. 12(a)). The significance of this contribution increases as the contour shrinks toward the crack tip. This contribution is evaluated by extending the last data available over this part of the contour on each side of the crack. Note that when out-of-plane motion is observed in the unloaded part of the sample, as in Fig. 6(d), the present computation has to be performed in the vicinity of the crack tip.

Results With the previous analysis, it is now possible to evaluate the possible domain independence of $\Phi$ by varying the radius of the integration contour for the steady-state crack growth. The corresponding results are presented in Figure 13 for the particular frame of Fig. 12(a). Fig. 13 indicates that the

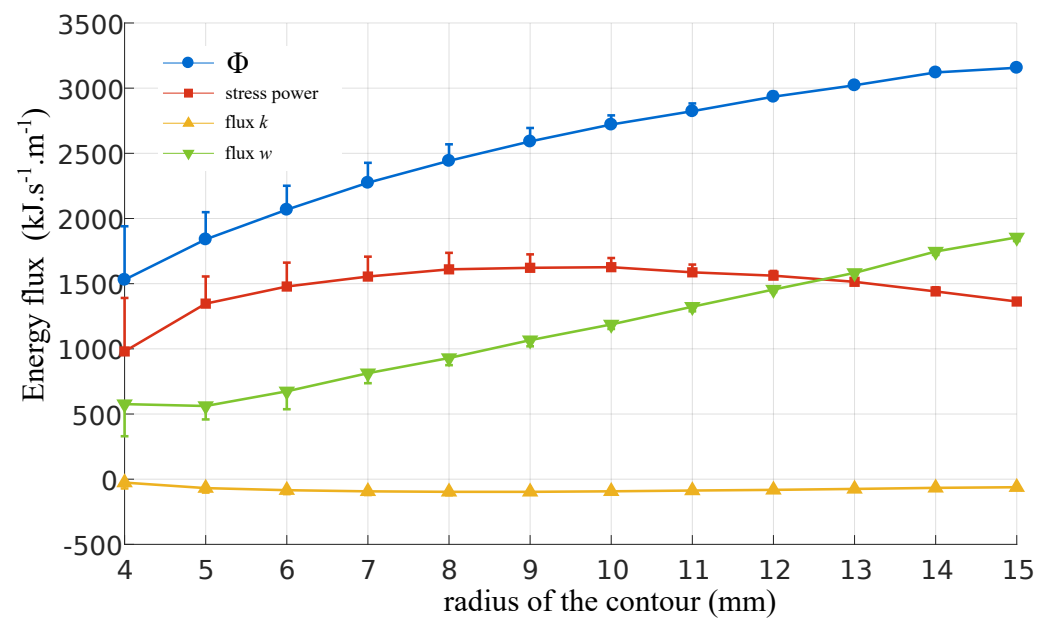

Fig. 13 Energy flux (Eq. 97) and its contributions vs. the contour radius. Error bars: values obtained with an estimate of the contributions close to crack lips.

energy flux is decreasing when the radius of the circle decreases, and therefore it is not path-independent. Note that considering the estimated contribution 
of parts of the contour close to the lips (error bars) reduces this decrease. The energy flux is made of three contributions (Eq. (9)): flux of strain energy, flux of kinetic energy, and the "flux across $\Sigma$ due to the material outside $\Sigma$ working on the material inside it" (Moran and Shih, 1987). The flux of kinetic energy is surprisingly small compared to the two other contributions; it is also negative as theoretically predicted by Gurtin and Yatomi (1980). Figure 14 illustrates the contributions of the integrand of Eq. (9) for a given contour. The use of circular contours suggests an angular description of the integrand,

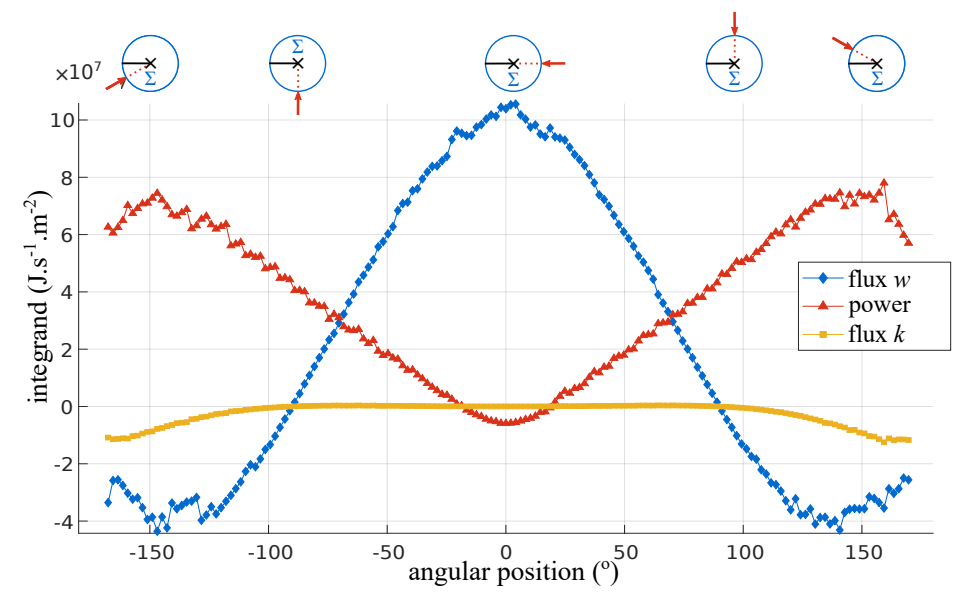

Fig. 14 Evolution of each contribution of the energy flux along a circular contour (radius $7 \mathrm{~mm}$ ). Small sketches show the positions along the contour with respect to the crack direction.

even if the interpretation as a direction of the fluxes obviously depends on the contour shape and has to be taken cautiously. Curves in Fig. 14 lead to a straightforward analysis of the origin of energy entering in $\Sigma$ : strain energy is mainly convected in front of the crack, kinetic energy is convected outside near the crack lips, and the power contribution increases behind the crack tip (area of high velocity and high shear strain). The kinetic energy contribution is comparable to the strain energy contribution behind the crack tip, as expected from their relative distribution shown in Fig. 10(c). However, the integration over the whole contour provides a small flux of kinetic energy compared to the other terms, in accordance with Fig. 13, The power contribution is the most important one close to the lips (around $\pm 150^{\circ}$ ), but its evolution toward crack lips $( \pm \pi)$ is not known due to the lack of data in these areas. Involving the velocity field, this contribution embodies the dynamic nature of the problem that the minor role played by the kinetic energy might understate.

Discussion The previous analysis demonstrates that it is possible to compute contour integrals in a dynamic and large strain context with current full field measurement techniques. Fields are sufficiently smooth for a trustworthy in- 
tegration on a contour a few millimetres away from the crack tip. However, the energy flux integral depends on the contour on which it is computed in the present steady-state crack growth case. Having checked the hypothesis of steady-state crack growth and estimated the flux close to the lips, two main reasons can explain this path-dependence. First, the constitutive model of the material does not take into account the influence of the strain rate, which is very significant especially in the area behind the crack tip as illustrated in Figure 15. Indeed, the strain rate varies from 0 to about $1500 \mathrm{~s}^{-1}$ and

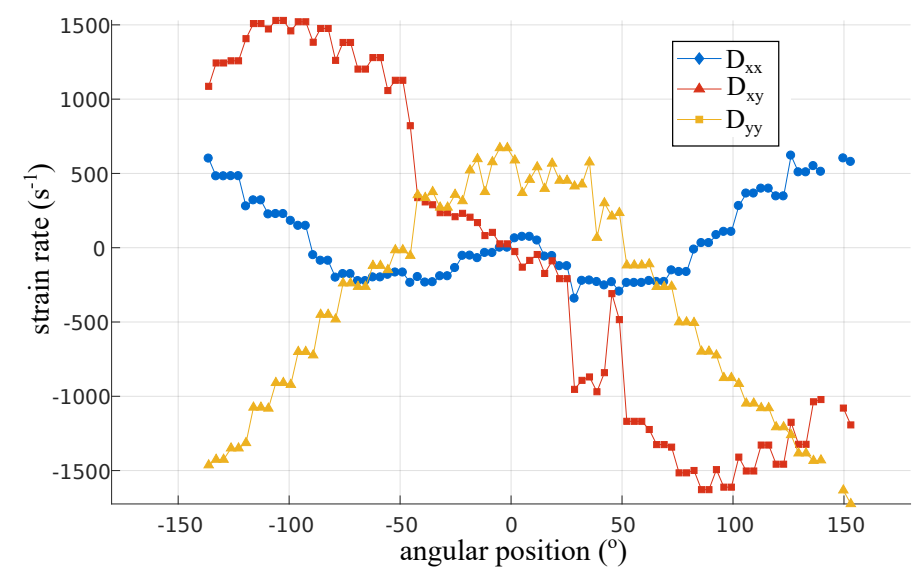

Fig. 15 Components of the strain rate tensor D (Eq. (8)) along the circular path of Fig. 14

such large strain rates induce a substantial stiffening of elastomer response (see for example Fig. 4 in Roland et al. (2007)). Therefore, the present constitutive equation probably underestimates the stress in the area behind the crack, reducing the power contribution in the energy flux. Second, due to the viscoelastic nature of elastomers, some viscous dissipation takes place in this area; it also relates to highly variable strain rates. Located far from the crack tip, this dissipation can occur outside some of the contours considered, explaining some of the decrease in energy flux as the contour radius decreases. To conclude, and contrary to the case of brittle gels where the path independence of the $J$-integral was experimentally retrieved by Livne et al. (2010), our quasi-static hyperelastic model may not be sufficient to precisely compute the dynamic energy release rate of elastomer. However, being able to identify a strain rate dependent model for strain rates varying from 0 to $1500 \mathrm{~s}^{-1}$ remains a difficult challenge.

4.2 Observing mechanical waves during crack growth

Quite surprisingly, the precision of the velocity field measurements offers another application to our set-up: observation of mechanical waves during crack 
growth. Indeed, the velocity field highlights the small displacement of the material points situated away from the crack tip, revealing the mechanical waves travelling across the sample as illustrated in Figure 16. The top picture of

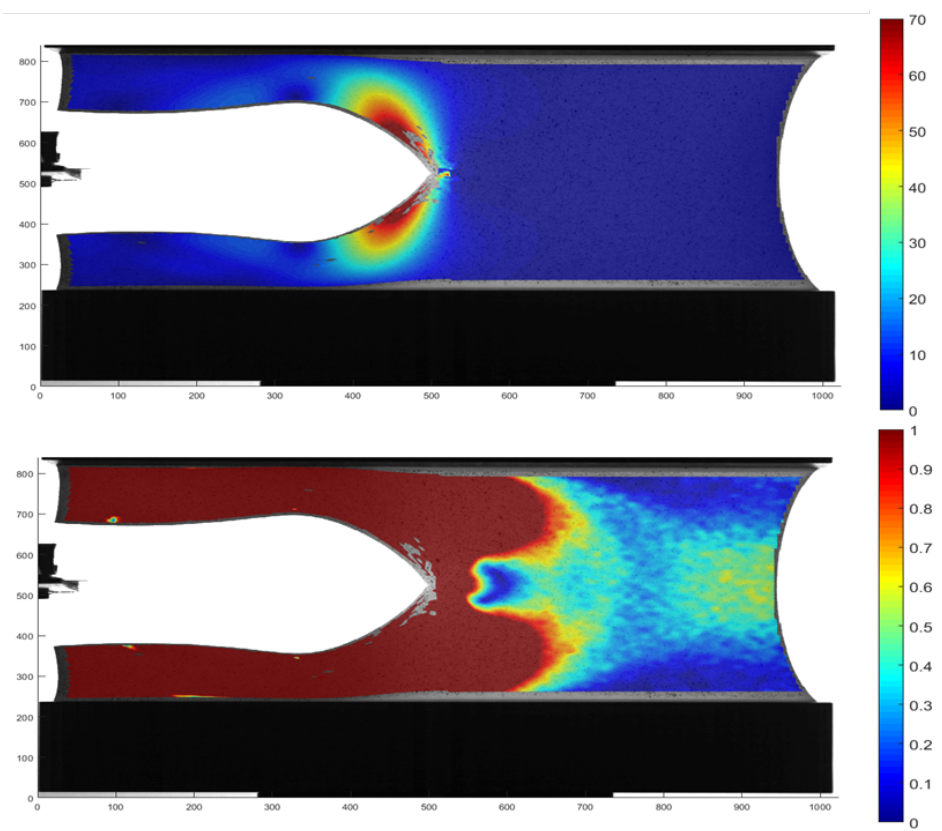

Fig. 16 Norm of the velocity field $\left(\mathrm{m} . \mathrm{s}^{-1}\right)$ superimposed on the picture from the HS camera. Top: full scale, 0 to $70 \mathrm{~m} \cdot \mathrm{s}^{-1}$. Bottom: reduced scale, 0 to $1 \mathrm{~m} \cdot \mathrm{s}^{-1}$. Prescribed stretch ratio: 3 - crack speed: $51 . \mathrm{s}^{-1}$.

Fig 16 presents the velocity norm in full scale, underlying the high retraction speed of the membrane right beyond the crack. This is the expected picture of the velocity field in this context, with a stationary pattern in the case of constant crack growth speed (Chen et al. 2011). As the velocity field is measured with a precision of about $0.05 \mathrm{~m} . \mathrm{s}^{-1}$, it is legitimate to reduce the scale between 0 and $1 \mathrm{~m} \cdot \mathrm{s}^{-1}$ as depicted in the bottom picture of Fig. 16 . It reveals some of the complex phenomena occurring in front of the crack tip (see supplementary videos). In a non-exhaustive manner, it exhibits:

- the influence of the material retraction ahead of the crack tip with possible wave reflection on the clamped edges (velocity larger than $1 \mathrm{~m} \cdot \mathrm{s}^{-1}$ close to the clamped edges ahead of the tip),

- the wave propagation along the horizontal axis of the specimen and reflection at the free edge (velocity up to $0.6 \mathrm{~m} \cdot \mathrm{s}^{-1}$ at the free edge),

- a point of zero velocity in front of the crack tip due to a change in the velocity sign along the horizontal axis: negative horizontal velocity close to the tip and positive far away. 
The negative velocity along the direction of propagation and close to crack tip is always observed, as noticeable in Fig 8 (a). Indeed, in front of the crack, the material is in a state of deformation close to uniaxial tension whereas it is closer to pure shear far away (Long et al. 2011). With both Poisson effect and incompressibility of the material, the strain in the horizontal direction is large enough to induce a measurable movement of material points towards the tip, hence a measurable velocity. This effect is particularly noticeable at the beginning of the test when the crack is initiated with a blade ; the sudden appearance of the crack induces this low velocity in the initially steady membrane as shown in Figure 17(a). The propagation of this pulse of negative
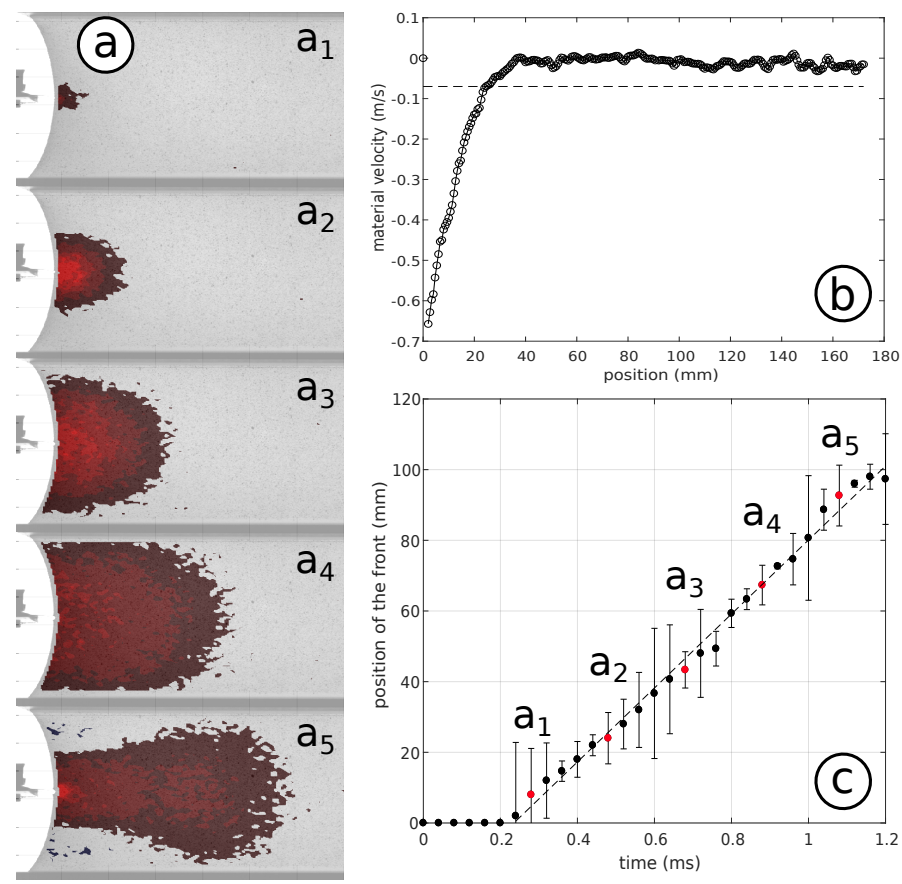

Fig. $17\left(a_{1}-a_{5}\right)$ Propagation of the pulse - horizontal velocity field just after the cutting (velocity less than $0.05 \mathrm{~m} . \mathrm{s}^{-1}$ in norm is blanked for clarity). (b) Shape of the pulse horizontal velocity along the centreline of the sample in picture $a_{2}$. Dotted line represents the threshold of $-0.07 \mathrm{~m} . \mathrm{s}^{-1}$. (c) Position of the front - position on the centreline where the threshold value is reached versus time; dotted line shows the linear fit giving a pulse speed of $105 \mathrm{~m} \cdot \mathrm{s}^{-1}$.

velocity is observed over a few images in some tests (HS camera frequency over $20000 \mathrm{~Hz}$ ). This pulse of horizontal displacement propagating along the horizontal direction corresponds to a longitudinal wave, and its speed can be measured by following a threshold value of the velocity as plotted in Fig. 17(b). In the case taken as example (prescribed stretch ratio of 3 ), the pulse propagates at about $105 \mathrm{~m} . \mathrm{s}^{-1}$ (Fig. 17(c)) for a threshold of $-0.07 \mathrm{~m} . \mathrm{s}^{-1}$ (the 
minimum speed detectable confidently given the uncertainty of $\pm 0.05 \mathrm{~m} . \mathrm{s}^{-1}$ at such low values). The velocity strongly depends on the threshold chosen, retrieving the dispersion of wave propagation in elastomers (Mason, 1963), and the value of $105 \mathrm{~m} . \mathrm{s}^{-1}$ is the maximum observed for this prescribed stretch ratio. Similar results could be obtained using directly the displacement field, highlighting even more the dispersion phenomenon. However, the temporal derivative increases the sensitivity which helps the definition of a clear threshold.

Analysing the small-scale displacement and velocity fields provides useful information to understand crack motion. The importance of waves is yet recognized in the case of brittle fracture (Goldman et al., 2010), and their influence on both crack speed and path in the particular case of large strain has to be confirmed. The longitudinal wave speed provides a reference value to characterise the crack speed. For instance, cracks in elastomer can grow faster than the shear wave speed but slower than the longitudinal wave speed: it corresponds to a propagation regime referred to as "intersonic" by Petersan et al. (2004). This value strongly depends on the anisotropy induced by stretching; it is therefore difficult to compute it analytically. Petersan et al. (2004) used a specific apparatus to measure it with a time-of-flight method. Our set-up provides a direct way to measure it, with an equivalent "optical" time-of-flight method. Even without the crack growth step, a similar set-up might offer the possibility to investigate the wave speed dependence on both its direction (induced anisotropy) and its amplitude.

\section{Conclusion}

The two-camera full-field technique proposed in this study offers broad new possibilities in the understanding of fracture mechanisms in materials under large strain. Crack growth can be observed in a very general framework, involving dynamic, large strain, and material non-linearities. Besides the mechanical framework, the set-up permits relaxing the hypotheses that have previously governed the study of dynamic cracks in elastomer membranes, such as the use of particular simple specimens. The use of DIC during the loading phase of the sample provides the membrane strain field in which the crack will grow, rendering possible complex crack paths and not only straight propagation at constant speed. Full-field measurements during crack growth also promise to bridge the gap between theoretical developments and experimental observations and measurements. Even if a local approach would require a more precise measurement technique at crack tip (Long and Hui, 2015), the energetic approach of fracture, from the direct energy balance to the complex $J$-integral formula, is well-suited to an experimental comparison. For instance, the controversy as to the use of the pure shear analytical formula in dynamic problems (Chen et al. 2011) advocates a closer look at crack behaviour through fullfield measurements. In spite of the strict validation of theoretical predictions, 
very general information, yet not easily obtainable, are available such as the longitudinal wave speed.

\section{Supplementary material}

Four short videos are proposed to illustrates some of the results presented in the paper.

- Crack_growth_slow_×700.avi presents the film recorded by the HS camera before any DIC treatment; its actual duration is about $12 \mathrm{~ms}$. It corresponds to Fig. 3 .

- Strain_reference_configuration.avi presents the evolution of two components of the Green Lagrange strain tensor during crack growth, in the material configuration. It corresponds to Fig 9 .

- Speed_field.avi shows the norm of the velocity vector, first in full scale then in a reduced one in order to reveal the complex phenomena during dynamic crack growth. It corresponds to Fig, 16 .

- Wave_horizontal_speed.avi is the film from which is extracted Fig 17 (a).

\section{Conflict of interest}

The authors declare that they have no conflict of interest.

\section{References}

Atkinson, C., Eshelby, J.D., 1968. The flow of energy into the tip of a moving crack. International Journal of Fracture Mechanics 4, 3-8.

Caimmi, F., Calabrò, R., Briatico-Vangosa, F., Marano, C., Rink, M., 2015. Jintegral from full field kinematic data for natural rubber compounds. Strain 51, 343-356.

Chao, Y.J., Luo, P.F., Kalthoff, J.F., 1998. An experimental study of the deformation fields around a propagating crack tip. Experimental mechanics 38, 79-85.

Chen, C.H., Zhang, H.P., Niemczura, J., Ravi-Chandar, K., Marder, M., 2011. Scaling of crack propagation in rubber sheets. EPL (Europhysics Letters) 96, 36009 .

Chevalier, L., Calloch, S., Hild, F., Marco, Y., 2001. Digital image correlation used to analyze the multiaxial behavior of rubber-like materials. European Journal of Mechanics-A/Solids 20, 169-187.

Dally, J.W., 1979. Dynamic photoelastic studies of fracture. Experimental Mechanics 19, 349-361.

Deegan, R.D., Petersan, P.J., Marder, M., Swinney, H.L., 2001. Oscillating fracture paths in rubber. Physical Review Letters 88, 014304. 
Freund, L.B., 1972. Energy flux into the tip of an extending crack in an elastic solid. Journal of Elasticity 2, 341-349.

Freund, L.B., 1998. Dynamic Fracture Mechanics. Cambridge University Press.

Gent, A.N., 1996. Adhesion and strength of viscoelastic solids. is there a relationship between adhesion and bulk properties? Langmuir 12, 44924496.

Gent, A.N., Marteny, P., 1982. Crack velocities in natural rubber. Journal of Materials Science 17, 2955-2960.

Goldman, T., Livne, A., Fineberg, J., 2010. Acquisition of inertia by a moving crack. Physical Review Letters 104, 114301.

Goldman Boué, T., Harpaz, R., Fineberg, J., Bouchbinder, E., 2015. Failing softly: a fracture theory of highly-deformable materials. Soft Matter 11, $3812-3821$.

Greensmith, H.W., 1963. Rupture of rubber. X. The change in stored energy on making a small cut in a test piece held in simple extension. Journal of Applied Polymer Science 7, 993-1002.

Greensmith, H.W., Thomas, A.G., 1955. Rupture of rubber. III. Determination of tear properties. Journal of Polymer Science Part A: Polymer Chemistry 18, 189-200.

Griffith, A.A., 1921. The phenomena of rupture and flow in solids. Philosophical Transaction of the Royal Society Series A, 221, 163-198.

Gurtin, M.E., Yatomi, C., 1980. On the energy release rate in elastodynamic crack propagation. Archive for Rational Mechanics and Analysis 74, 231247.

Kadir, A., Thomas, A.G., 1981. Tear behavior of rubbers over a wide range of rates. Rubber Chemistry and Technology 54, 15-23.

Kirugulige, M.S., Tippur, H., 2009. Measurement of fracture parameters for a mixed-mode crack driven by stress waves using image correlation technique and high-speed digital photography. Strain 45, 108-122.

Lake, G.J., Lawrence, C.C., Thomas, A.G., 2000. High-speed fracture of elastomers: Part I. Rubber Chemistry and Technology 73, 801-817.

Livne, A., Bouchbinder, E., Svetlizky, I., Fineberg, J., 2010. The near-tip fields of fast cracks. Science 327, 1359-1363.

Long, R., Hui, C.Y., 2015. Crack tip fields in soft elastic solids subjected to large quasi-static deformation - a review. Extreme Mechanics Letters 4, $131-155$.

Long, R., Krishnan, V.R., Hui, C.Y., 2011. Finite strain analysis of crack tip fields in incompressible hyperelastic solids loaded in plane stress. Journal of the Mechanics and Physics of Solids 59, 672-695.

Marckmann, G., Verron, E., 2006. Comparison of hyperelastic models for rubber-like materials. Rubber Chemistry and Technology 79, 835-858.

Marder, M., 2006. Supersonic rupture of rubber. Journal of the Mechanics and Physics of Solids 54, 491-532.

Mason, P., 1963. Finite elastic wave propagation in rubber. Proc. R. Soc. Lond. A 272, 315-330. 
Maugin, G.A., 1993. Material inhomogeneities in elasticity. volume 3. CRC Press.

Maugin, G.A., 1995. Material forces: concepts and applications. Appl. Mech. Rev. 48,

Moran, B., Shih, C.F., 1987. Crack tip and associated domain integrals from momentum and energy balance. Engineering Fracture Mechanics 27, $615-$ 642.

Morishita, Y., Tsunoda, K., Urayama, K., 2016. Velocity transition in the crack growth dynamics of filled elastomers: Contributions of nonlinear viscoelasticity. Physical Review E 93, 043001.

Moulinet, S., Adda-Bedia, M., 2015. Popping balloons: A case study of dynamical fragmentation. Physical Review Letters 115, 184301.

Ogden, R.W., 1972. Large deformation isotropic elasticity-on the correlation of theory and experiment for incompressible rubberlike solids, in: Proc. R. Soc. Lond. A, 326, 565-584.

Petersan, P.J., Deegan, R.D., Marder, M., Swinney, H.L., 2004. Cracks in rubber under tension exceed the shear wave speed. Physical Review Letters 93, 015504.

Petiteau, J.-C., Othman, R., Guégan, P., Le Sourne, H., Verron, E., 2013a. Dynamic uniaxial extension of elastomers at constant true strain rate. Polymer Testing 32, 394-401.

Qi, Y., Zou, Z., Xiao, J. Long, R., 2019. Mapping the nonlinear crack tip deformation field in soft elastomer with a particle tracking method. Journal of the Mechanics and Physics of Solids 125, 326-346.

Rice, J.R., 1968. A path independent integral and the approximate analysis of strain concentration by notches and cracks. Journal of Applied Mechanics $35,379-386$.

Rivlin, R.S., Thomas, A.G., 1953. Rupture of rubber. I. Characteristic energy for tearing. Journal of Polymer Science 10, 291.

Roland, C.M., Twigg, J.N., Vu, Y., Mott, P.H., 2007. High strain rate mechanical behavior of polyurea. Polymer 48, 574-578.

Rublon, P., Huneau, B., Verron, E., Saintier, N., Beurrot, S., Leygue, A., Mocuta, C., Thiaudière, D., Berghezan, D., 2014. Multiaxial deformation and strain-induced crystallization around a fatigue crack in natural rubber. Engineering Fracture Mechanics 123, 59-69.

Stevenson, A., Thomas, A.G., 1979. On the bursting of a balloon. Journal of Physics D: Applied Physics 12, 2101.

Sutton,M.A. Orteu, JJ. Schreier, H., 2009. Image correlation for shape, motion and deformation measurements: basic concepts, theory and applications. Springer Science \& Business Media.

Treloar, L.R.G., 1944. Strains in an inflated rubber sheet, and the mechanism of bursting. Rubber Chemistry and Technology 17, 957-967.

Treloar, L.R.G., 1944. Stress-strain data for vulcanised rubber under various types of deformation. Transactions of the Faraday Society 40, 59-77.

Washabaugh, P.D., Knauss, W.G., 1994. A reconciliation of dynamic crack velocity and Rayleigh wave speed in isotropic brittle solids. International 
Journal of Fracture 65, 97-114.

Yeoh, O.H., 2001. Analysis of deformation and fracture of 'pure shear' rubber testpiece. Plastics, Rubber and Composites 30, 389-397.

Zhang, H.P., Niemczura, J., Dennis, G., Ravi-Chandar, K., Marder, M., 2009.

Toughening effect of strain-induced crystallites in natural rubber. Physical Review Letters 102, 245503. 